\title{
Le sarcome de Kaposi au cours du SIDA : cancer ou hyperplasie ?
}

Le sarcome de Kaposi frappe environ $20 \%$ des malades qui meurent de SIDA, presque exclusivement des hommes dans les pays développés. L'étude de cette prolifération avait été jusqu'alors très entravée par l'absence d'un système de culture des cellules constituant les sarcomes de Kaposi, avant tout des éléments fusiformes assez caractéristiques de l'affection et qui sont mélangés à d'autres types cellulaires au sein d'un tissu richement vascularisé.

L'équipe de Robert Gallo vient de parvenir à stimuler la prolifération de cette population cellulaire grâce à l'utilisation d'un milieu conditionné par les produits que libèrent des lymphocytes $\mathrm{T} \mathrm{CD}^{+}{ }^{+}$infectés par les virus leucémogènes HTLV 1 ou 2, ou par les virus du SIDA HIV l et 2 .

Outre des facteurs de croissance connus (interleukine l, TNF, etc.), les lymphocytes infectés produiraient un nouveau type de facteur de croissance, stimulant spécifiquement la prolifération des cellules fusiformes des sarcomes de Kaposi, recrutées à partir de cellules endothéliales. Ces éléments fusiformes ainsi stimulés sécréteraient à leur tour divers facteurs de croissance qui seraient responsables de l'angiogenèse $\left(\mathrm{m} / \mathrm{s} n^{\circ} 5\right.$, vol. 4, p. 318) et du recrutement de nouveaux types cellulaires. Ainsi la prolifération du sarcome de Kaposi serait-elle secondaire à une réponse « hyperplasique » de cellules normales stimulées par des facteurs de croissance libérés par les lymphocytes $\mathrm{T}$ infectés et agissant selon un mode paracrine. Les facteurs de croissance produits à leur tour par les cellules stimulées agiraient de façon paracrine (et peut-être également autocrine) pour amplifier l'hyperplasie [1, 2].

Selon une autre équipe, dirigée par G. Jay (travaillant comme R. Gallo au NCI de Bethesda, MD, USA), le sarcome de Kaposi doit plutôt être considéré comme une prolifération cancéreuse secondaire à l'expression d'un gène transformant le gène tat [3].

Des souris transgéniques mâles exprimant le gène tat sous le contrôle du promoteur et du enhancer du LTR de HIV-l développent des lésions cutanées ressemblant d'assez près au sarcome de Kaposi. Alors que les mâles et les femelles accumulent la même quantité de messagers HIV. tat dans leurs cellules cutanées, seuls les mâles ont, dans $15 \%$ des cas, des proliférations de type Kaposi, suggérant l'intervention d'un facteur hormonal dans le déclenchement des tumeurs. Curieusement, le messager n'est plus détectable dans les cellules tumorales [3]. Le gène tat code pour une protéine qui active en trans l'expression du génome viral. Il pourrait donc aussi agir sur des gènes cellulaires dont la modulation serait à l'origine du développement tumoral. D'autres protéines virales transactivatrices ont cette même propriété (la protéine ElA d'adénovirus, par exemple). La protéine Tat du virus leucémogène HTLV-1 provoque, quant à elle, des tumeurs neurofibromateuses chez les souris transgéniques exprimant une construction HTLV-l/tat $\left(\mathrm{m} / \mathrm{s} n^{\circ} 10\right.$, vol. 3 , p. 623).

Ainsi les deux groupes du NCI convergent-ils pour faire du sarcome de Kaposi une conséquence de l'infection par le virus HIV, conséquence directe pour le groupe de Jay, qui considère que la protéine Tat est responsable de la transformation, hyperplasie secondaire à la sécrétion de facteurs de croissance par les lymphocytes infectés pour le groupe de Gallo. L'absence de messager Tat dans les tumeurs de Kaposi semble plus en faveur de cette dernière thèse que de celle de Jay. La protéine Tat pourrait peut-être intervenir dans la libération de facteurs de croissance par les cellules exprimant le transgène. Il faut noter d'ailleurs qu'une prolifération secondaire à une hyperlibération de facteurs de croissance agissant de façon paracrine ou autocrine peut aussi être parfois considérée comme une cancérisation... ; c'est le mécanisme impliqué dans la transformation par l'oncogène $v$-sis, codant pour un équivalent de la chaîne B du PDGF (platelet derived growth factor).

A. $\mathbf{K}$.

1. Nakamura S, Salahuddin SZ, Biberfeld P, et al. Kaposi's Sarcoma cells : long-term culture with growth factor from retrovirus-infected CD4 ${ }^{+}$T cells. Science $1988 ; 242: 426-30$. 2. Salahuddin SZ, Nakamura S, Biberfeld $P$, et al. Angiogenic properties of Kaposi's sarcoma-derived cells after long-term culture in vitro. Science $1988 ; 242: 430-3$

3. Vogel J, Hinrichs SH, Reynolds RK, Luciw PA, Jay G. The HIV tat gene induces dermal lesions resembling Kaposi's sarcoma in transgenic mice. Nature 1988 ; 335 : 606-11. 